\title{
Identificación de regiones expuestas a bajas temperaturas en el Perú usando imágenes de la temperatura de la superficie del suelo procedente de sensor MODIS/Aqua
}

\author{
Jaime Aguilar-Lome y Joel Rojas Acuña \\ Facultad de Ciencias Físicas, Universidad Nacional Mayor de San Marcos, Lima, Perú. \\ Recibido el 19 de junio del 2018. Aceptado el 5 de julio del 2018
}

DOI: https://doi.org/10.33017/RevECIPeru2018.0007/

\section{Resumen}

La temperatura de la superficie terrestre (LST, siglas en inglés) es una variable clave en las interacciones y los flujos de energía entre la superficie de la Tierra y la atmósfera. Se ha analizado la LST MODIS nocturna a una resolución espacial de $1 \mathrm{~km}$ en el periodo 2003-2017 (junio-agosto) sobre el Perú, para identificar las regiones expuestas a bajas temperaturas. Nuestro resultado muestra que las regiones por debajo de los $0^{\circ} \mathrm{C}$ se encuentran por encima de $3500 \mathrm{msnm}$ (en promedio). A demás la LST nocturna promedio mensual está correlacionado con la temperatura mínima media mensual del aire $(R=0.96, N=763)$ y la topografía influye significativamente en la variabilidad de la LST.

Descriptores: LST MODIS, Heladas Radiativas, Andes.

\section{Abstract}

Land Surface Temperature (LST) is a key variable in the interactions and energy fluxes between the Earth surface and the atmosphere. The MODIS LST nighttime at spatial resolution of $1 \mathrm{~km}$ was analyzed during the period 2003-2017 (June-August) over Peru to identify regions exposed to low temperatures. Our result shows that the regions below $0^{\circ} \mathrm{C}$ are above 3500 masl (in average). In addition, the mean monthly nighttime LST is correlated with the mean monthly minimum air temperature $(R=0.96, N=763)$ and the topography significantly influences the variability of LST.

Keywords: MODIS LST, Radiation Frost, Andes.

\section{Introducción}

Los fenómenos extremos son los que mayor impacto producen en las sociedades y en la economía de los pueblos [1]. Las heladas son parte de eventos extremos, consideradas como fenómenos meteorológicos capaces de afectar el rendimiento, la supervivencia o la calidad de las personas, animales y cultivos, siendo los mayores riesgos asociados a la producción agropecuaria [2]. En Perú, principalmente la sierra sur, es afectada por el recrudecimiento de fenómenos climáticos y microclimáticos extremos (nevadas, heladas, granizadas, vientos y friajes) que afectan a la población, medios de vida, recursos naturales y servicios esenciales, incidiendo en deterioro de las condiciones de vida y pobreza en la población [3, 4]. El análisis estadístico de datos meteorológicos, ha permitido conocer las características climatológicas de las heladas en la sierra de Perú: fechas promedio y extremas para la primera y última helada, los períodos libres de helada, tanto de tipo meteorológico como de tipo agronómico, para diferentes umbrales de temperatura crítica de los cultivos [5]. Sin embargo, los resultados no podrían ser satisfactorios a partir de observaciones puntuales, cuando las mediciones no están disponibles en densidad suficiente. La teledetección por satélite, al tener acceso a toda la cubierta terrestre (océanos y continentes), permite 
obtener datos homogéneos y realizar análisis multitemporales [2], convirtiéndose hoy en día, en una de las más importantes fuentes de información climática en el mundo. Por ejemplo, con la información captada por los sensores térmicos de los satélites NOAA-AVHRR (National Oceanic and Atmospheric Administration-Advanced Very High Resolution Radiomete), zonificaron áreas con diferentes niveles de riesgo de ocurrencia de heladas en Altiplano boliviano [6], en la Provincia de Entre Ríos [7] y en la región Pampeana (Argentina) [8]. También integrando la temperatura de la superficie del suelo (o Land Surface Temperature, LST, en inglés) medida por el sensor MODIS/Aqua durante noche con los descriptores fisiográficos (latitud, altitud, distancia a los salares, pendiente, insolación potencial, convergencia topográfica, etc.), se generó el mapeo de los riesgos de helada en el Altiplano sur de Bolivia [9]. El Instituto Geofísico del Perú (IGP), determinó que, en ciertas zonas de valle de Mantaro, la temperatura del aire menor a $0^{\circ} \mathrm{C}$ tiene mayor incidencia durante los meses entre junio y agosto con un máximo en julio, asociados a poca cobertura nubosa, escasa humedad atmosférica y escasa humedad del suelo [10]. El objetivo del presente trabajo es identificar las regiones del Perú expuestas a bajas temperaturas empleando los datos de la LST MODIS/Aqua nocturna (LSTn) durante el periodo invierno austral (junio, julio y agosto) de 2003 a 2017.

\section{2. Área de estudio y datos}

El área de estudio comprende el territorio peruano. El Perú posee una gran diversidad de climas (27 de 32 existentes en el mundo), debido a la presencia de la Cordillera de los Andes, la Corriente de Humboldt y la Corriente del Niño, que choca en las costas de Piura y Tumbes; a estos accidentes se suma el Anticiclón del Pacífico Sur [11]. Su clima varía de acuerdo a cada región geográfica. Así, en la sierra varía de templado-cálido hasta helado. En los niveles intermedios de la cordillera (entre 2,500 y $3,500 \mathrm{msnm}$ ), la temperatura anual promedio varía entre $11.0^{\circ} \mathrm{C}$ y $16.0^{\circ} \mathrm{C}$. Las precipitaciones varían en diferentes escalas y tienen una marcada estacionalidad, con un pico entre enero y marzo, mientras que de mayo a agosto la precipitación es baja y la insolación fuerte $[12,13]$.

En este trabajo se utiliza el producto LST MODIS (Moderate Resolution Imaging Spectroradiometer) (MYD11A2, versión 6) obtenida del sensor MODISAqua en su paso nocturno entre las 01:30 horas y las 03:00 horas (hora local) [14], por ser más cercanas a las temperaturas mínimas del aire registradas aproximadamente a las 6 a.m. en las estaciones meteorológicas. El producto MYD11A2 es un compuesto cada 8 días de los valores diarios de la LST (MYD11A1) a $1 \mathrm{~km}$ de resolución espacial. Este producto involucra la LST diurna (13:30-15:30 horas) y nocturna, y su correspondiente información de calidad de las estimaciones de la LST [15]. El primer producto de LST MODIS es recuperado de la temperatura de brillo usando el algoritmo generalizado split window propuesto por Wan y Dozier [16] únicamente en condiciones de una atmósfera libre de nubes, mediante la siguiente ecuación:

$$
\begin{aligned}
& \mathrm{T}_{S}=\mathrm{C}+\left(\mathrm{B}_{1}+\mathrm{B}_{2} \frac{1-\varepsilon}{\varepsilon}+\mathrm{B}_{3} \frac{\Delta \varepsilon}{\varepsilon^{2}}\right) \frac{\mathrm{T}_{31}+\mathrm{T}_{32}}{2}+ \\
& \quad\left(\mathrm{A}_{1}+\mathrm{A}_{2} \frac{1-\varepsilon}{\varepsilon} \mathrm{A}_{3} \frac{\Delta \varepsilon}{\varepsilon^{2}}\right) \frac{\mathrm{T}_{31}-\mathrm{T}_{32}}{2} \\
& \varepsilon=0.5\left(\varepsilon_{31}+\varepsilon_{32}\right) \\
& \Delta \varepsilon=\left(\varepsilon_{31}-\varepsilon_{32}\right)
\end{aligned}
$$

donde $T_{31} y T_{32}$, son las temperaturas de brillo en la banda 31 y $32 ; \varepsilon_{31}$ y $\varepsilon_{32}$ son las emisividades de la superficie del suelo en estas dos bandas estimada usando el método basado en clasificación de emisividad $[17,18]$ según al tipo de cobertura de suelo determinado del producto de cobertura de suelo (MOD12Q1) y de producto de cobertura de nieve (MOD10_L2). Los coeficientes $A_{i}(i=$ $1,2,3), B_{i}(i=1,2,3)$ y $C$ son dados por interpolación de un conjunto de datos multidimensional de tablas de consulta.

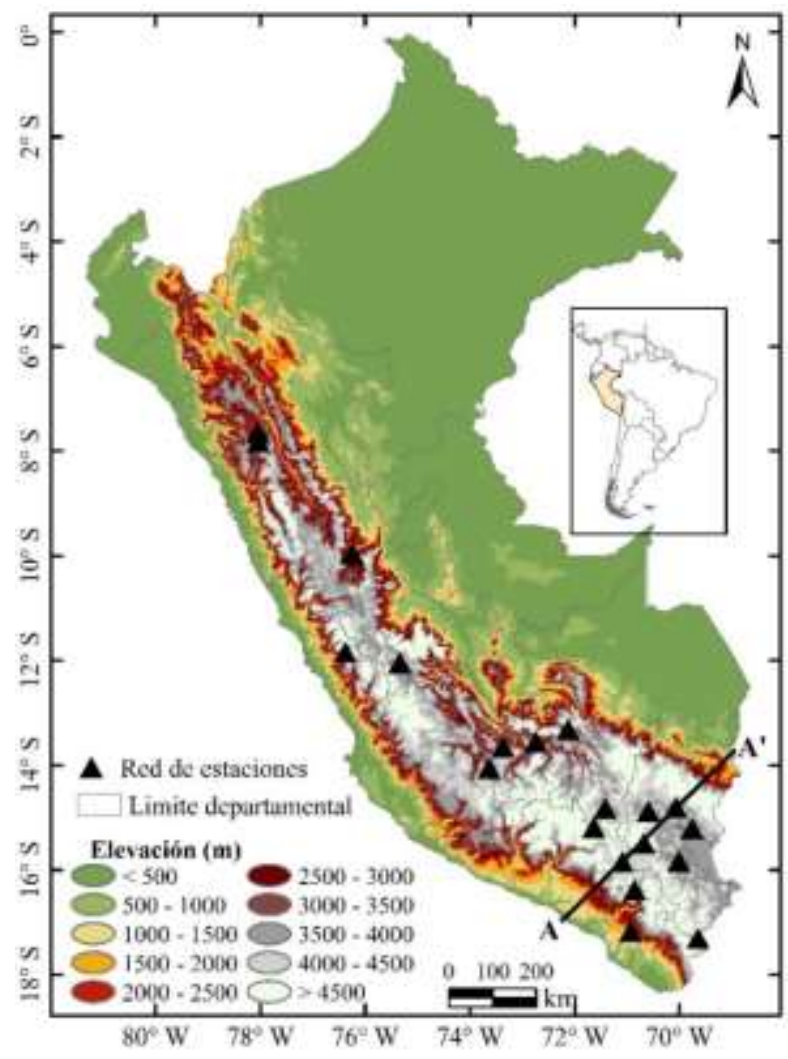


Figura1: Mapa de elevación del área de estudio. Los triángulos en color negro, representan la ubicación de estaciones meteorológicas.

Para representar las formas del relieve del área de estudio, utilizamos el modelo de elevación digital (MED) GTOPO30 (Global Topographic Data). GTOPO30 se basa en los datos derivados de 8 fuentes de información sobre la elevación, incluyendo vectores y conjunto de imágenes MEDs. El espaciado de grilla es 30 segundos de arco $(0.008333333$ grados o $1 \mathrm{~km})$ [19]. Mientras el conjunto de datos de temperatura mínima media mensual del aire fueron proporcionados por Servicio Nacional de Meteorología e Hidrología (SENAMHI)-Perú. Que consiste de 2003 a 2015 (junio-agosto) observado en 20 estaciones meteorológicas distribuidas sobre los 1000 msnm.

\section{Metodología}

Tabla 1 resume la información de la calidad de datos del producto LSTn sobre el área de estudio, obtenida al evaluar mediante las imágenes de fiabilidad (QC). Se define datos de buena calidad, los que tienen un error promedio para emisividad $\leq$ 0.01 y para LST $\leq 0.01 K$ [20]. Si no se logran estas precisiones para las estimaciones de LST y emisividad, el píxel se clasifica con otro valor de banda de calidad. En nuestro caso los pixeles con error promedio de LST $>2 \mathrm{~K}$ y emisividad $>0.02$ no son considerados datos válidos.

Tabla 1: Frecuencia de calidad de datos observados durante el periodo 2003-2017 (junioagosto). $Q C=$ Control de calidad. EP=Error promedio.

\begin{tabular}{|l|l|l|}
\hline $\begin{array}{l}\text { QC } \\
\text { (0-255) }\end{array}$ & Descripción & LSTn \\
\hline 0 & LST de buena calidad & $66.50 \%$ \\
\hline 2 & LST no calculado & $17.67 \%$ \\
\hline 17 & $\begin{array}{l}\text { LST con EP } \leq 1 K \text { y y } \\
\text { emisividad con EP } \leq 0.02\end{array}$ & $3.55 \%$ \\
\hline 65 & $\begin{array}{l}\text { LST con EP } \leq 2 K \quad \text { y } \\
\text { emisividad con EP } \leq 0.01\end{array}$ & $10.96 \%$ \\
\hline 81 & $\begin{array}{l}\text { LST con EP } \leq 2 K \text { y y } \\
\text { emisividad con EP } \leq 0.02\end{array}$ & $1.30 \%$ \\
\hline
\end{tabular}

Una vez que se realizan estas correcciones de la LSTn mediante su control de calidad, se calcularon los promedios mensuales. Los datos de IFOV (campo de visión instantáneo) en grados Kelvin $(K)$ se convirtieron en grados Celsius $\left({ }^{\circ} \mathrm{C}\right)$. A partir de esta información, se seleccionaron 20 áreas de
$3 \mathrm{~km} \times 3 \mathrm{~km}$ con centro en cada una de las estaciones meteorológicas, siguiendo la metodología desarrollada por François y otros [6]. De esta manera, se obtuvo un promedio de los 9 pixeles de la LSTn, posteriormente fueron evaluadas la relación mediante regresión lineal simple con la temperatura mínima media mensual del aire, durante el periodo 2003-2015 (junioagosto). Como bondad de ajuste del modelo se ha utilizado el coeficiente de correlación de Pearson y como medida del error el RMSE (error cuadrático media).

\section{Resultados y discusión}

En la Figura 2 se muestra la distribución de la LSTn, para la serie de tiempo que corresponde al periodo 2003-2017 (junio- agosto), para pixeles espaciales de $1 \times 1 \mathrm{~km}$. En la Figura 2 se observa los rangos de temperatura consideradas para el análisis. Las temperaturas por debajo de los $0^{\circ} \mathrm{C}$ corresponden a las zonas con mayor elevación en las regiones de Ancash, Huánuco, Cerro de Pasco, Junín, Huancavelica, Ayacucho, Apurímac, Cusco, Puno, Arequipa, Moquegua y Tacna. También se puede apreciar que en los alrededores del lago Titicaca, la LSTn promedio está por encima de los $0^{\circ} \mathrm{C}$. Este hecho se debe a que el agua tiene mayor capacidad calorífica respecto a la superficie del suelo en la época estudiada. Durante las noches claras o cuando el cielo está libre de la presencia de nubes, la pérdida de energía de la superficie por emisión de radiación de onda larga se acentúa, provocando una caída de temperatura del aire próximo a la superficie del suelo, resultando en lo que se denomina inversión térmica [5], dando origen a la llamada heladas por radiación. También en los estudios realizados en Altiplano boliviano señalan que, en latitudes bajas, con mayor elevación y en áreas de escasa vegetación; las bajas temperaturas se asocian debido a la pérdida de energía radiante de la superficie del suelo, durante las noches despejadas, con aire de poca humedad $[6,9]$.

En la Figura 3 se presenta el ajuste de regresión entre la LSTn promedio mensual y la temperatura mínima media mensual del aire de un total de 20 estaciones meteorológicas ubicadas sobre 1000 msnm con coeficiente de correlación de 0.96. El grado de ajuste obtenido fue similar al hallado por otros autores [9] en la región Altiplano boliviano, donde los datos de temperatura mínima del aire diarios registrada en tres estaciones meteorológicas y la LSTn están altamente correlacionado $(R=0.90, N=750)$. Esto les permitió generar mapas regionales con una resolución de 
$1 \mathrm{~km}$ mostrando las variaciones de riesgo de helada en tres periodos sucesivos en la época de cultivo.

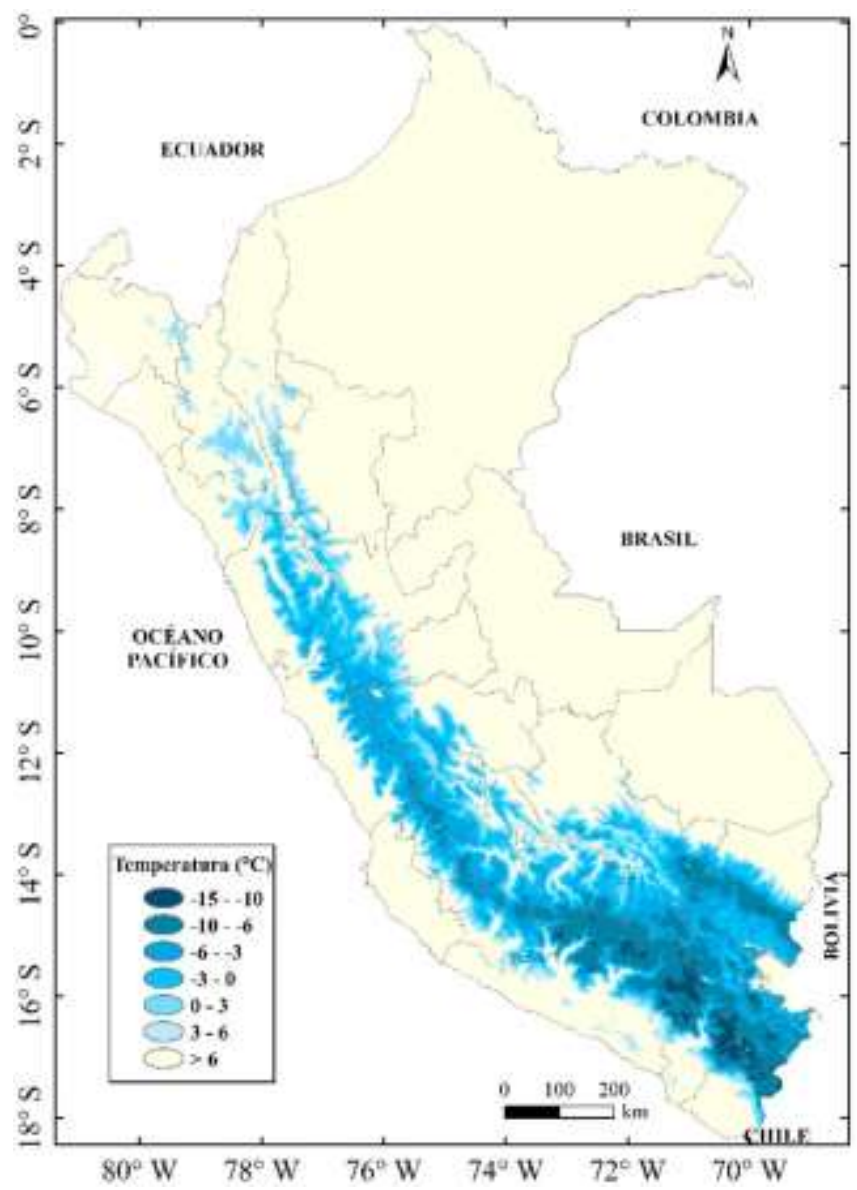

Figura 2: Temperatura de la superficie del suelo nocturna promedio. Periodo 2003-2017(junioagosto).

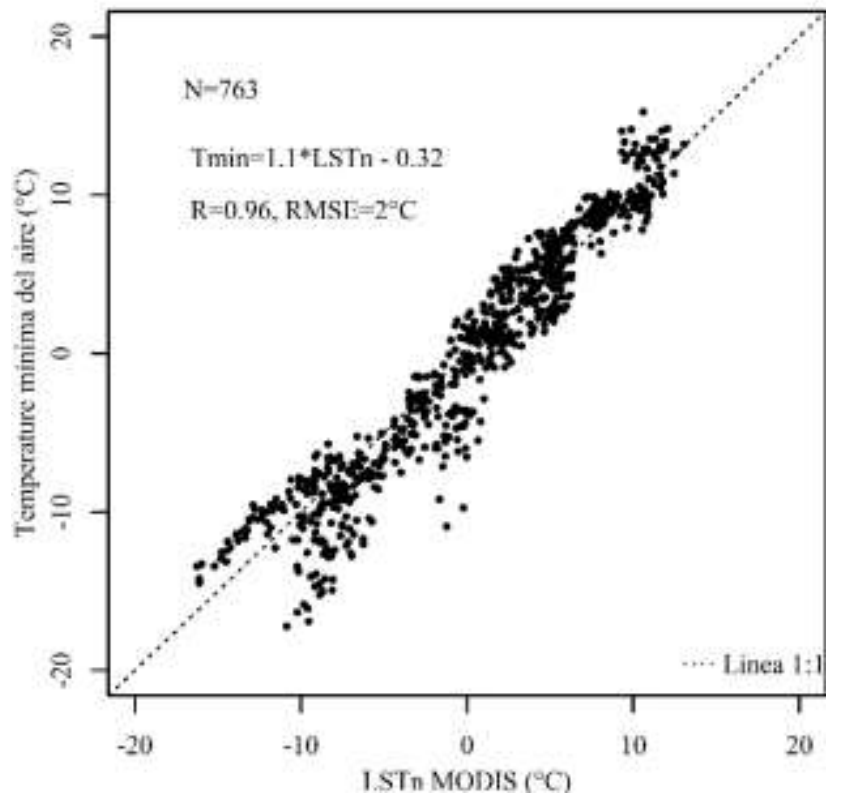

Figura 3: Regresión lineal entre la temperatura de la superficie del suelo nocturna promedio mensual y la temperatura mínima media mensual del aire durante el periodo 2003-2015 (junio-agosto).
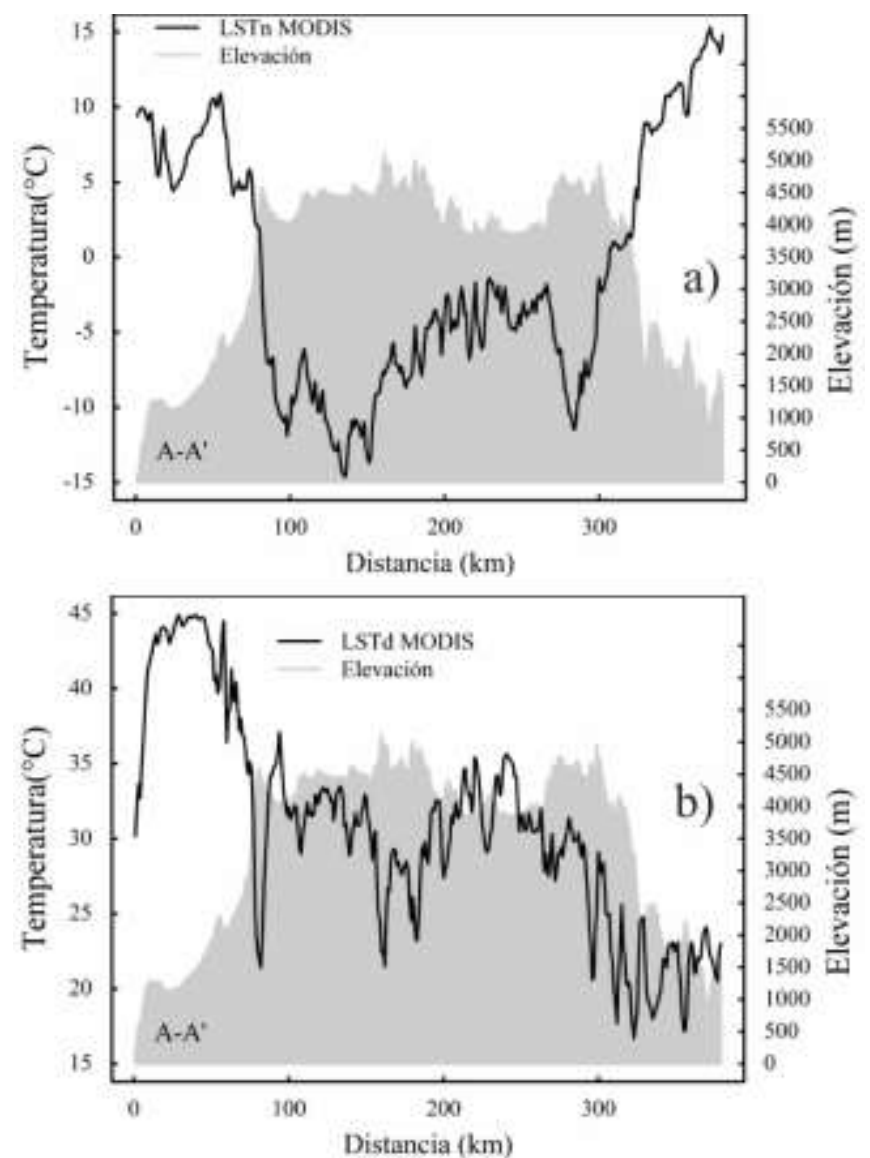

Figura 4: Variación de la temperatura de la superficie del suelo a) nocturna (promedio junioagosto) y b) diurna (promedio junio-agosto) con la elevación.

Tabla 2: Coeficiente de determinación entre la elevación y el promedio mensual de la LST diurna y nocturna, obtenidas en el transecto $A-A$ '.

\begin{tabular}{|c|c|c|c|c|}
\hline$R^{2}$ & Junio & Julio & Agosto & Junio-agosto \\
\hline $\begin{array}{c}\text { LTS } \\
\text { día }\end{array}$ & 0.12 & 0.11 & 0.05 & 0.10 \\
\hline $\begin{array}{c}\text { LST } \\
\text { noche }\end{array}$ & 0.78 & 0.75 & 0.81 & 0.79 \\
\hline
\end{tabular}

En la Figura 4 (a) se muestra un transecto que se extiende de oeste a este a través de la parte sur del Perú. El mayor descenso de la LSTn se produce en la zona de transición entre la costa y la sierra. La LSTn por debajo de $0^{\circ} \mathrm{C}$, se observa por encima de $3500 \mathrm{msnm}$, a unos $80 \mathrm{~km}$ desde la línea de la costa. En la ladera oriental por debajo de 4500 msnm, la LSTn comienza a aumentar progresivamente con la disminución de la altura, 
hasta alcanzar $15^{\circ} \mathrm{C}$ sobre $1000 \mathrm{msnm}$. La figura 4(b) muestra la variación de la LST diurna (junioagosto) con la elevación. Los transectos (Figura 4(a) y 4(b)) muestran que la heterogeneidad de la cobertura terrestre genera cambios drásticos de la LST diurna y nocturna, siendo mucho más variable la LST diurna. La LST diurna (Figura 4(b)) presenta valores altos en áreas desérticas (costa peruana) y disminuye a medida que acerca a la presencia de la cobertura vegetal (selva peruana). Este proceso es debido a que el alto contenido de humedad $\mathrm{y} / \mathrm{o}$ presencia de cobertura vegetal, permite que una mayor fracción del flujo neto de radiación sea balanceado por la evapotranspiración y por el flujo de calor latente, disminuyendo de esta manera el flujo de calor sensible y por lo tanto la LST diurna [22]. Mientras durante la noche en ausencia de la radiación solar este proceso es nula [21; 23]. Para cuantificar el grado de relación de la LST diurna y nocturna con la elevación, se calculó el coeficiente de determinación. De la tabla 2 podemos inferir que la variabilidad de la LST nocturna promedio (junioagosto) en el transecto A-A' es explicada en $79 \%$ por la topografía. Según Bertoldi y otros [21], en las áreas montañosa, la LST está controlada por una interacción compleja de topografía, radiación solar entrante y procesos atmosféricos, así como la distribución de humedad del suelo, diferentes tipos de cobertura y tipos de vegetación.

\section{Conclusiones}

El análisis de regresión mostró que existe una alta correlación positiva entre el promedio mensual de la LST de noche de MODIS-Aqua y la temperatura mínima media mensual del aire en el periodo 20032015 (junio-agosto), esto permitió representar a través de un mapa zonas de ocurrencia de heladas en el Perú. Las regiones con temperatura por debajo de $0^{\circ} \mathrm{C}$ se encuentran por encima de 3000 msnm. La topografía (altitud) influye significativamente en la variabilidad de la LST, aunque la correlación varía según el mes del año.

\section{Referencias}

[1] G.V. Müller. Variabilidad interanual de las heladas en la Pampa húmeda. Revista Brasilera de Meteorología, 21(1) (2006) 135-141.

[2] J.V. Straschnoy et al. Caracterización espacial del estrés hídrico y de las heladas en la región pampeana a partir de información satelital y complementaria. RIA. Revista de
Investigaciones

Agropecuarias, 35(2) (2006).

[3] E. Moya, J. Torres Guevara. Familias alpaqueras enfrentando el cambio climático. In $m$ (No. L01 M93. Soluciones Prácticas-ITDG, 2008) Lima (Perú).

[4] G R PUNO-CRGP. Plan Regional de Gestión del Riesgo de Desastres 20162021 (2016).

[5] FAO (Organización de la Naciones Unidas para la Agricultura y la Alimentación), Atlas de heladas del Perú, Convenio de Cooperación Técnica Interinstitucional, SENAMHI-FAO (Lima, Perú, 2010).

[6] C. François, R. Bosseno, J.J. Vacher, B. Seguin. Frost risk mapping derived from satellite and surface data over the Bolivian Altiplano. Agricultural and forest meteorology, 95(2) (2009) 113-137.

[7] C. Di Bella, et al. Frost mapping in Entre Rios Province using satellite images, Revista Brasileira de Agrometeorologia, Santa Maria, 5 (1997) 269-274.

[8] H. Kerdiles, M. Grondona, R. Rodriguez, B. Seguin. Frost mapping using NOAA AVHRR data in the Pampean region, Argentina. Agricultural and Forest Meteorology, 79(3) (1996) 157-182.

[9] R. Pouteau et al. Downscaling MODISderived maps using GIS and boosted regression trees: the case of frost occurrence over the arid Andean highlands of Bolivia. Remote Sensing of Environment, 115(1) (2011) 117-129.

[10] IGP (Instituto Geofísico del Perú). Vulnerabilidad y adaptación al cambio climático en la cuenca del río Mantaro, (Vol. III, Fondo Editorial CONAM, Lima, Perú, 2005)

[11] SENAMHI. Mapa de Clasificación Climática del Perú. Método de Thornthwaite, (Eds. Senamhi Perú, 1988).

[12] SENAMHI. Escenario de cambio climático en la Cuenca del río Mantaro para el año 2100, (Lima, Perú, 2011).

[13] J.C. Espinoza Villar et al. Spatio-temporal rainfall variability in the Amazon basin countries (Brazil, Peru, Bolivia, Colombia, and Ecuador). International Journal of Climatology, 29(11) (2009) 1574-1594.

[14] F. Marzban, T. Conrad, P. Marzban, S. Sodoudi. Estimation of the Near-Surface Air Temperature during the Day and Nighttime from MODIS in Berlin, Germany. International Journal of 
Advanced Remote Sensing and GIS, (2018).

[15] Z. Wan. MODIS land surface temperature products users' guide, (ERI, University of California, Santa Barbara, 2013).

[16] Z. Wan, J. Dozier. A generalized Splitwindow algorithm for retrieving landsurface temperature from space, IEEE Transaction on Geoscience and Remote Sensing, 34(4) (1996) 892-905.

[17] W.C. Snyder, Z. Wan, Y. Zhang, Y. Z. Feng. Classification-based emissivity for land surface temperature measurement from space, International Journal of Remote Sensing, 19(14) (1998) 27532774.

[18] W.C. Snyder, Z. Wan. BRDF models to predict spectral reflectance and emissivity in the thermal infrared. IEEE Transactions on Geoscience and remote Sensing, 36(1) (1998) 214-225.

[19] https://ta.cr.usgs.gov/GTOPO30
[20] Z. Wan, Y. Zhang, Q. Zhang, Z. Li. Quality assessment and validation of the MODIS global land surface temperatura, International Journal of Remote Sensing, 25 (2004) 261-274.

[21] G. Bertoldi et al. Topographical and ecohydrological controls on land surface temperature in an alpine catchment. Ecohydrology, 3(2) (2010) 189204.

[22] B. Douseet, F. Goumelon. Satellite multisensor data analysis of urban surface temperaturas and landcover. ISPRS Journal of Photogrammetry \& Remote Sensing, 58 (2003) 43-54.

[23] D. Stroppiana, et al. Seasonality of MODIS LST over Southern Italy and correlation with land cover, topography and solar radiation. European Journal of Remote Sensing, 47(1) (2004) 133-152.

E-mail: jaguilarlome@gmail.com 